Musées, Patrimoine et Culture scientifiques et techniques

$184 \mid 2019$

juillet-août 2019

\title{
Voir la lumière : Patrimoine et culture scientifique en milieu scolaire
}

Anne-Sophie Rozay et Sylvain Chambreland

\section{OpenEdition}

\section{Journals}

Édition électronique

URL : http://journals.openedition.org/ocim/2926

DOI : 10.4000/ocim.2926

ISSN : 2108-646X

Éditeur

OCIM

Édition imprimée

Date de publication : 1 juillet 2019

Pagination : 32-37

ISSN : 0994-1908

Référence électronique

Anne-Sophie Rozay et Sylvain Chambreland, « Voir la lumière : Patrimoine et culture scientifique en milieu scolaire », La Lettre de I'OCIM [En ligne], 184 | 2019, mis en ligne le 01 juillet 2020, consulté le 25 janvier 2021. URL : http://journals.openedition.org/ocim/2926 ; DOI : https://doi.org/10.4000/ocim 2926

Ce document a été généré automatiquement le 25 janvier 2021.

Tous droits réservés 


\section{Voir la lumière : Patrimoine et culture scientifique en milieu scolaire}

Anne-Sophie Rozay et Sylvain Chambreland

Bouquet de lampes, collection de l'université de Rouen

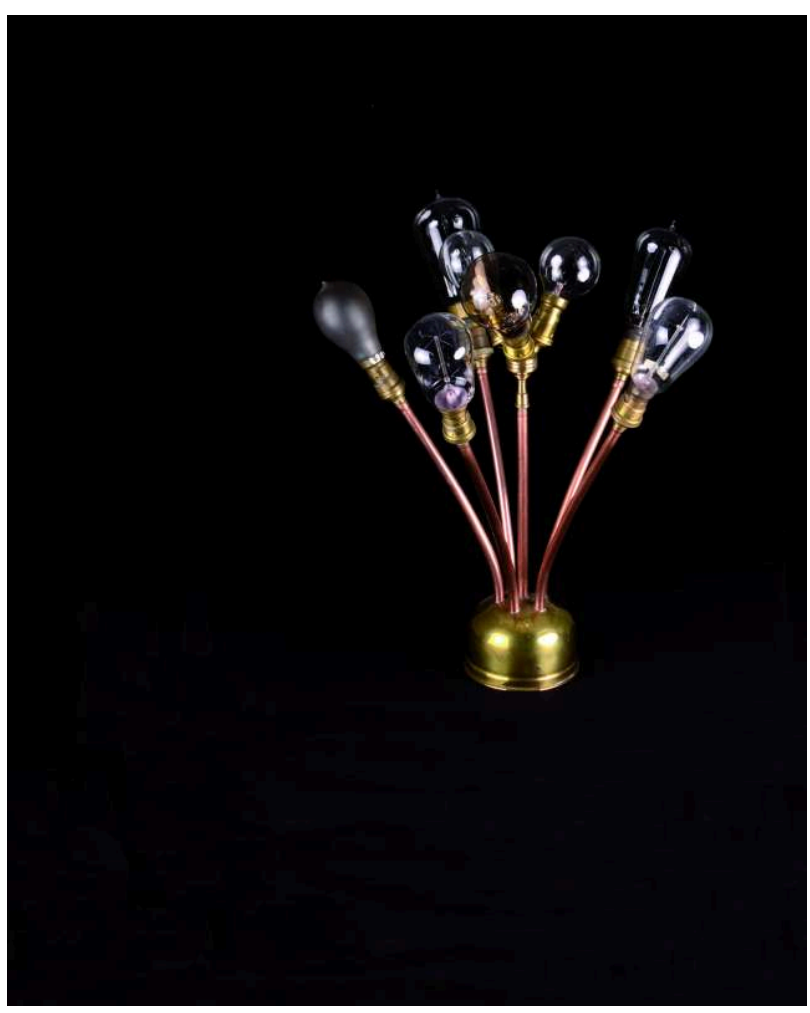

(C) Vincent Guibert 
1 Dans tout projet d'action culturelle, la question de la mobilisation des publics est un enjeu fondamental. Capter le public scolaire pose un certain nombre de contraintes, surtout lorsque celui-ci est géographiquement éloigné des lieux de diffusion culturelle. Le réseau Résitech, en partenariat avec le service culturel de la Communauté de communes Lyons-Andelle, a initié un projet d'itinérance d'exposition dans deux collèges implantés en zone rurale.

2 Si la mise en place d'ateliers de découverte, et/ou artistique avec l'implication de collectivités n'est pas nouvelle ${ }^{1}$, l'originalité du projet tient sans doute dans l'exploitation du patrimoine scientifique comme outil culturel et pédagogique, mais aussi dans l'échelle territoriale. Il est à noter le rôle important des bénévoles du réseau Résitech tant dans les relations avec les collectivités territoriales que dans la médiation sur le terrain.

\section{Historique du projet}

3 Le réseau Résitech mène depuis 2008 des actions de valorisation des collections du patrimoine scientifique issues de l'enseignement supérieur et de laboratoires de recherche. Science Action Normandie, un des deux centres de Culture Scientifique, Technique et Industrielle (CCSTI) en Normandie, siège au comité de pilotage du réseau Résitech, et des collaborations ponctuelles sont fréquentes².

4 L'idée d'une exposition commune est née lors du lancement de l'évènement « 2015 année internationale de la lumière et des techniques utilisant la lumière $»^{3}$. Ce sujet présentait un double intérêt : d'un point de vue patrimonial, il permettait de valoriser une partie des collections d'instruments en optique et, d'un point de vue culturel, cette thématique offrait l'occasion d'aborder un grand nombre de questions transversales : qu'est-ce que la lumière ? Comment la produit-on ? Lumière naturelle et lumière artificielle ? Comment la mesure-t-on ? Quelles sont ses propriétés?

5 La coproduction de l'exposition s'est répartie de la manière suivante : Science Action Normandie apportait un soutien financier et son expertise en matière de diffusion de culture scientifique, tandis que Résitech prenait en charge le commissariat de l'exposition et sa mise en œuvre.

Ce partenariat avec Science Action Normandie impliquait que cette exposition soit intégrée au catalogue des expositions itinérantes du CCSTI.

7 Le concept d'exposition itinérante affiche en théorie de nombreux aspects positifs. Cependant, dans la pratique le fait que cette exposition soit composée principalement d'objets patrimoniaux complique le cahier des charges, en ajoutant notamment des contraintes de transport et sécuritét. Un scénographe (Étienne David) a été approché pour concevoir un mobilier dédié répondant aux différentes contraintes techniques.

\section{Le réseau Résitech}

Depuis 2008, le réseau Résitech (Réseau Scientifique Industriel et Technique HautNormand) œuvre en Normandie pour la sauvegarde et la valorisation du patrimoine scientifique et technique. Porté par l'Insa Rouen Normandie et l'université de Rouen Normandie, ce réseau est né suite au partenariat entre l'Insa et la Mission nationale de sauvegarde du patrimoine scientifique et technique 
contemporain du Cnam-Musée des Arts et Métiers (Patstec). Le réseau Résitech rassemble aujourd'hui des établissements d'enseignement supérieur, laboratoires de recherche, musées, associations et des collectivités.

Le programme de sauvegarde préconisé par la Mission nationale Patstec implique la réalisation d'un inventaire et la valorisation de ce patrimoine. Ainsi, le réseau s'inscrit régulièrement dans des opérations d'actions culturelles en participant notamment à des opérations nationales, comme la Fête de la science, ou encore les Journées du Patrimoine.

Parallèlement, le réseau initie ses propres actions au travers de projets pouvant prendre différentes formes comme par exemple, accueillir des classes pour des visites ponctuelles de réserves d'instruments, ou encore se déplacer dans les classes pour sensibiliser les élèves à la sauvegarde du patrimoine scientifique au moyen d'ateliers pédagogiques.

Parallèlement, le réseau propose trois expositions itinérantes Les objets des sciences (2008), La science d'hier à aujourd'hui, une histoire d'instrument ! (2012) et Voir la lumière? Mesure, science et curiosité (2015).

\section{Diffusion de l'exposition}

8 En 2015, l'exposition Voir la lumière, mesure, sciences et curiosité est présentée une première fois lors de la Fête de la science. Elle a par la suite été présentée au Pôle Régional des Savoirs de Rouen à l'automne 2016. Ce dernier lieu comportait plusieurs points attractifs : une salle d'exposition, une localisation proche du centre ville à proximité d'une station de tramway. Cependant, ce lieu n'étant pas identifié habituellement comme lieu d'exposition, et malgré la communication mise en œuvre peu de public s'est déplacé.

9 Prenant note de cet échec partiel, une réflexion a été entamée pour mettre en place de nouvelles stratégies notamment pour impliquer les publics scolaires.

Dans le cadre du conseil scientifique du réseau, un élu d'une commune de l'Eure a proposé de présenter l'exposition et ses potentialités pédagogiques au service culturel de la Communauté de Communes de Lyons-Andelle. Le public scolaire abordé habituellement par le rectorat et les enseignants, l'a été cette fois directement par les communes et la communauté de communes. Ce canal n'est donc pas à négliger : les communautés de communes entretiennent elles aussi des liens avec les établissements et peuvent être forces de proposition.

11 Située à une trentaine de kilomètres de Rouen, la Communauté de Communes de LyonsAndelle bénéficie d'équipements socioculturels et d'une programmation riche, mais ne dispose pas sur son territoire d'établissement de type muséal. La proposition de diffuser l'exposition constituait donc une offre originale et le projet a donc été favorablement accueilli. Deux collèges ont répondu positivement à la sollicitation du service culturel de la Communauté : le collège Guy de Maupassant de Fleury-surAndelle et le collège La Côte des Deux Amants de Romilly-sur-Andelle. 
Voir la lumière. Mesure, sciences et curiosité

Cette exposition coproduite avec Science Action Normandie a pour objectif d'aborder des propriétés de la lumière au prisme d'objets techniques patrimoniaux. Les thématiques présentées sont l'éclairage, l'optique dans l'enseignement et dans la recherche. Les objets exposés sont issus des collections patrimoniales de l'Insa Rouen Normandie, de l'université de Rouen Normandie, du laboratoire Coria (Complexe de Recherche Interdisciplinaire en Aérothermochimie), de l'Association de Sauvegarde du Patrimoine Électrique et Gazier (Aspeg) et de collections privées.

Souhaitant valoriser les aspects patrimoniaux de ces collections, la scénographie de l'exposition repose sur les codes de la muséographie classique : les objets et documents sont présentés sous vitrines, accompagnés de cartels descriptifs avec les titres, années et numéros d'inventaire, et de « fiches de salles » explicatives. Cette mise en scène globale n'est pas anodine puisqu'elle confère aux objets scientifiques un aspect patrimonial.

- un premier module présente des objets liés à l'éclairage, cette thématique permet d'aborder l'« effet Joule ", mais aussi la fluorescence jusqu'aux dernières avancées technologiques telles que la Li-Fi (Light Fidelity), une technologie de communication basée sur la lumière visible et exploitant la lumière des connexions internet ;

- un deuxième module se décline sur des objets liés cette fois aux propriétés de la lumière, les objets présentés abordent l'optique « simple »;

- le troisième module présente des objets issus d'un laboratoire de recherche : des lasers, et deux prototypes d'instruments de mesure de très hautes températures exploitant des propriétés optiques.

Afin de compléter ce contenu statique, il a semblé nécessaire d'insérer quelques dispositifs animés pour « montrer la lumière ». Le premier choix a été la diffusion d'une boucle vidéo présentant différents aspects de la lumière.

Trois autres vidéos réalisées spécifiquement pour l'exposition sont diffusées en boucle sur des tablettes. Réalisées sur un mode court et dynamique, ces vidéos illustrent des propriétés de la lumière exploitées dans la recherche avec de courtes interviews de chercheurs (« Agence Spectrum » Laurent Matthieu et Franck Herouard www.scienceaction.asso.fr/ressources/videos/voir-la-lumi\%C3\%A8reyakaphoton).

Enfin, un dernier dispositif réalisé par Jean Hoche, membre bénévole du réseau Résitech, propose une maquette reproduisant les phénomènes luminescents des aurores boréales. 
Vue de l'exposition Voir la lumière. Mesures, sciences et curiosité, Pôle Régional des Savoirs, Rouen 2016

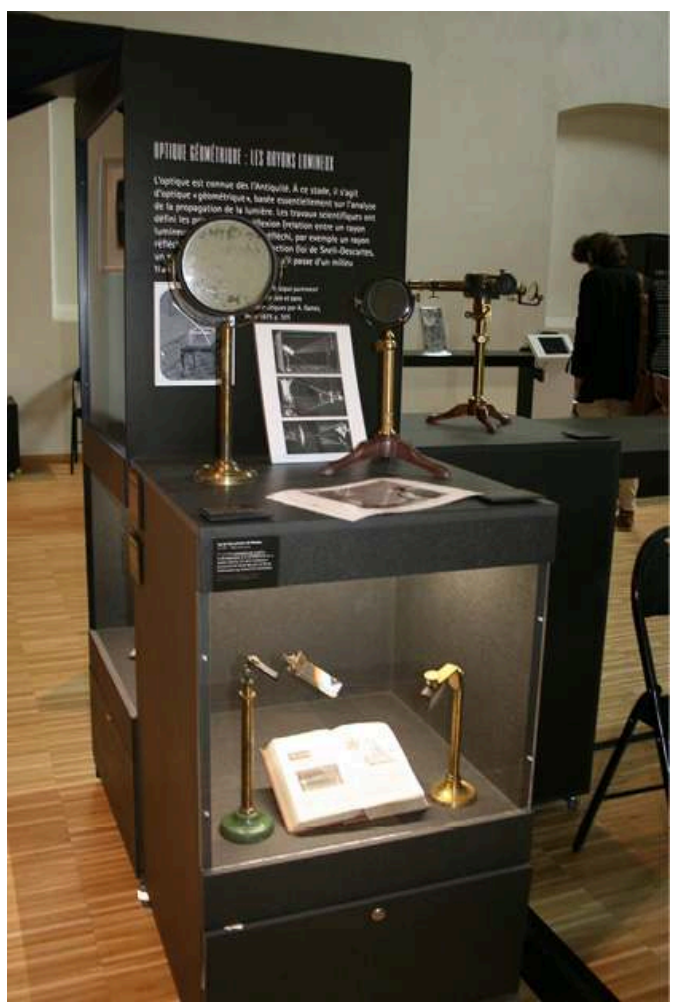

(c) Résitech

\section{Construction d'un projet pédagogique commun}

Pour une mise en œuvre pertinente, il est nécessaire de construire un projet cohérent pour tous les acteurs engagés. Après plusieurs rencontres entre les différents partenaires (le service culturel de la communauté de communes, les équipes pédagogiques des collèges et le réseau Résitech), deux axes ont été privilégiés :

- un temps de visite commentée de l'exposition Voir la lumière, assuré par un médiateur du réseau Résitech ;

14 - un temps " actif » sous la forme d'ateliers. Un collège a choisi de mettre en place un atelier sur la découverte de la spectroscopie. Encadré par deux enseignants du collège, l'atelier consistait entre autre à fabriquer un spectroscope. Dans le second établissement, un atelier « inventaire » du patrimoine a été mis en place. Après une initiation aux techniques et méthodologie d'inventaire, les élèves complétaient une fiche d'inventaire, puis présentaient oralement l'objet sur lequel ils avaient travaillé. 


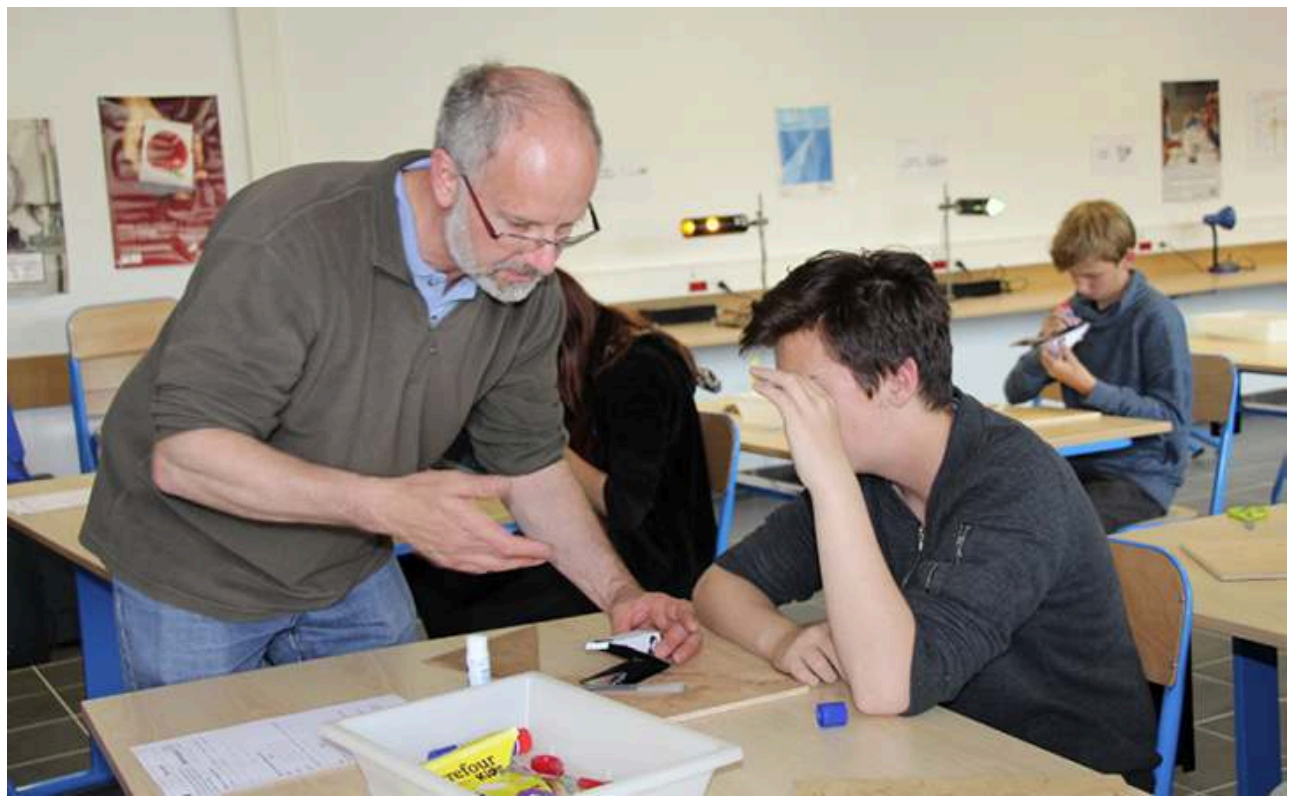

(c) Résitech

\section{La mise en œuvre du projet et le retour d'expérience}

En 2017, le collège de Fleury-sur-Andelle a accueilli l'exposition pendant une semaine. Toutes les classes de $6^{\mathrm{e}}, 4^{\mathrm{e}}$ et $3^{\mathrm{e}}$ ont bénéficié de ce dispositif. Puis en 2018 , c'est le collège de Romilly-sur-Andelle qui l'a reçue, en faisant participer les classes de $4^{\mathrm{e}}$ et $3^{\mathrm{e}}$. $\mathrm{Au}$ total 750 élèves ont ainsi pu bénéficier de cette action. Les classes ont été divisées en deux groupes, chaque groupe participant à une visite puis à un atelier. Les Principales des collèges ont banalisé des heures, ce qui illustre l'implication des équipes pédagogiques. L'installation de l'exposition dans les murs du collège $\mathrm{a}$ eu un fort impact sur le personnel et les élèves.

Concrètement, les élèves ont eu le sentiment qu'un «musée " s'était installé dans leurs murs, une occasion pour eux d'avoir un nouveau regard sur le patrimoine et l'histoire des techniques, mais aussi de découvrir les métiers du patrimoine.

La médiation humaine lors des visites permet d'établir un dialogue direct avec les élèves. Les échanges ont permis aux élèves de se « décomplexer " face à des appareils qui leur étaient souvent inconnus. Cette exposition a été également une occasion pour les élèves de découvrir l'existence de laboratoires de recherche sur un territoire proche de chez eux. Des personnes bénévoles du réseau ont également participé activement à la mise en place de l'exposition et, ponctuellement, à sa médiation, ce qui a enrichi humainement et scientifiquement le dispositif.

Cette opération entre dans les missions du réseau Résitech. Un budget annuellement dédié à ce type d'opération prévoit le temps de travail d'un emploi à temps plein, comprenant à la fois des missions d'inventaire des instruments de laboratoire et leur valorisation au travers de ce type de projet. La Communauté de Communes de l'Andelle a mis à disposition du personnel pour la manutention de l'exposition. 
Spectroscope fabriqué par Ducretet, fin XIX $\mathrm{X}^{\mathrm{e}}$-début $\mathrm{XX}$ e siècles, collection de l'université de Rouen.

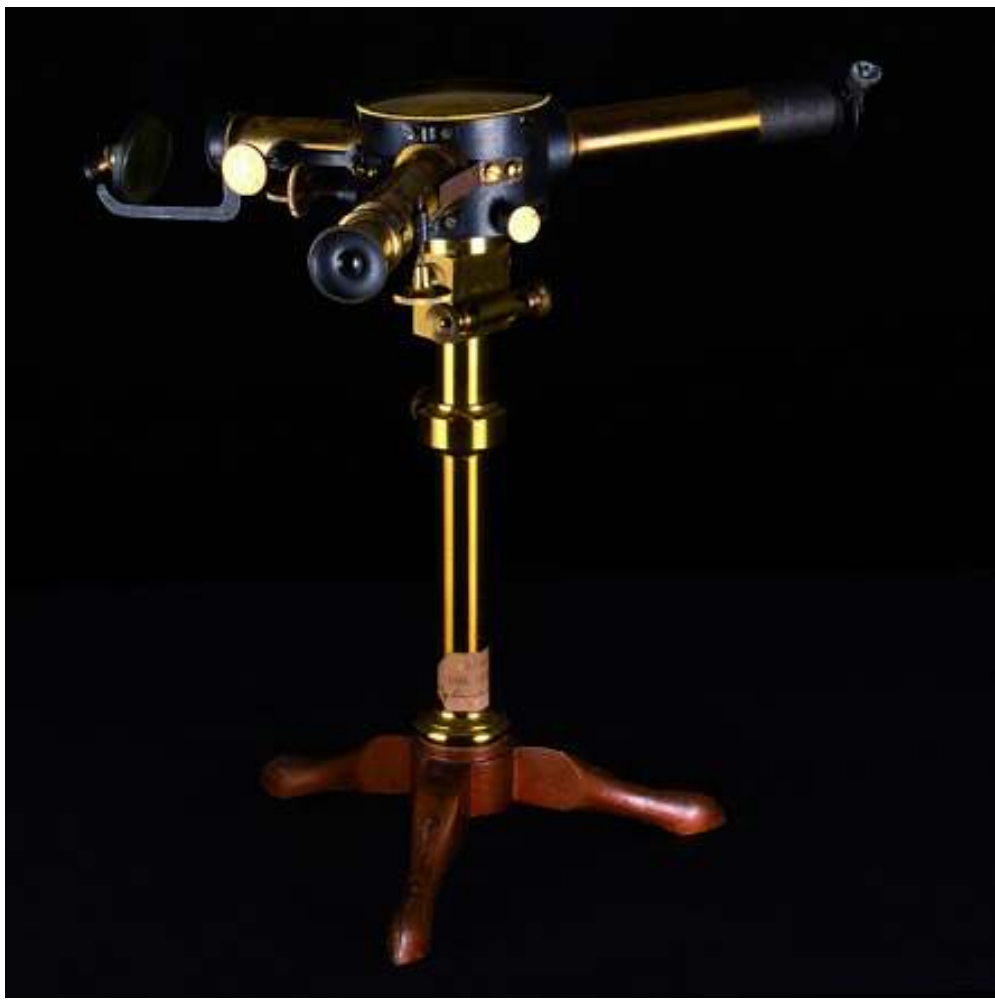

(C) Vincent Guibert

\section{Témoignage d'une enseignante}

La visite commentée de l'exposition a permis aux élèves d'être sensibilisés aux évolutions techniques de l'éclairage de la bougie à la LED en passant par la lampe électrique à incandescence. Ils se sont ensuite familiarisés aux principes fondamentaux de l'optique à travers la découverte des collections pédagogiques universitaires : lentilles, prismes et miroirs. Enfin, ils ont pu découvrir l'existence de laboratoires de recherche normands à travers des prototypes exploitant certaines propriétés de la lumière issus de ces laboratoires.

Les retours enthousiastes des élèves révèlent qu'ils ont été à la fois marqués par les objets scientifiques et par la découverte des métiers du patrimoine et de la recherche.

Dans un second temps, l'atelier a débuté par des observations de spectres lumineux pour expérimenter différents phénomènes liés à la perception des couleurs, puis sur la construction d'un spectroscope en carton. Cette expérience a fasciné tous les élèves de la $6^{\mathrm{e}}$ à la $3^{\mathrm{e}}:$ « c'est beau toutes ces couleurs! » dit une élève de $6^{\mathrm{e}}$, « on dirait l'arc en ciel » dit un autre en $4^{\mathrm{e}}$.

Cette approche a permis de faire comprendre aux élèves que la lumière qu'ils observaient, était composée de plusieurs radiations colorées. Bon nombre d'entre eux étaient très ravis de pouvoir emmener le spectroscope chez eux pour le montrer à leurs parents. 
En tant qu'enseignante, j'ai trouvé l'exposition enrichissante pour des élèves de collège et très accessible grâce à la visite commentée ponctuée d'anecdotes. Ils ont ainsi pu voir le lien qui existe entre les cours scientifiques et leur utilité dans les recherches d'aujourd'hui.

La fabrication du spectroscope n'est pas toujours simple avec les élèves, ils ont ainsi pu découvrir qu'ils pouvaient faire des expériences scientifiques avec $\mathrm{du}$ matériel issu de la vie quotidienne. Cette expérience serait à reconduire.

Par Carine Soulas (Professeur de Sciences de la Vie et de la Terre, collège Guy de Maupassant de Fleury-sur-Andelle)

\section{Quels bilans et quel avenir?}

Ce dispositif permet d'aborder des connaissances scientifiques par le prisme d'objets du quotidien, pédagogiques et de recherche. C'est donc une occasion d'approcher des principes physiques autrement que par une entrée strictement scientifique. L'exposition est à la fois un outil de vulgarisation scientifique, une porte d'entrée sur l'histoire des sciences et des enjeux des sciences et techniques dans la société.

Les objectifs pour Résitech ont été atteints puisque l'investissement reste raisonnable et les résultats en terme du nombre d'élèves impliqués tout à fait satisfaisants.

$\mathrm{Du}$ point de vue des collèges, les enseignants et responsables pédagogiques ont su s'approprier l'exposition et valoriser l'installation dans les murs des établissements scolaires comme un réel évènement. En outre, même si les retombées sont plus difficilement mesurables, cette initiative permet de travailler les compétences communicationnelles des élèves (présentation orale des spectroscopes fabriqués) et enfin, de vivre l'interdisciplinarité de manière concrète et pratique. Pour les enseignants, cette expérience est un outil complémentaire aux enseignements classiques. C'est également une occasion de renouveler les liens avec l'enseignement supérieur, milieu dont les enseignants sont issus, mais s'en sont souvent éloignés au fil du temps.

Enfin pour la Communauté de Communes Lyons-Andelle, c'est une manière de développer les liens sur le territoire. En organisant un vernissage à destination des élus locaux, les partenaires ont pu se rencontrer autour de thématiques nouvelles.

La réussite de ce dispositif réside en partie dans la mise en place d'une coordination collaborative où chaque partenaire a pu apporter ses spécificités et ses compétences.

Et après ? Le réseau Résitech n'a pas vocation à pérenniser ce type de projet, mais pourrait être partenaire actif si d'autres acteurs s'emparaient de ce dispositif.

Les auteurs tiennent à remercier Daniel Allano, Sophie Avonde, Annabel Beaupied, Catherine Boivin, Anne Caldin, Joël Cordier, Claire Grelle, Frédéric Hamelin, Jean Hoche, Jean-Noël Le Toulouzan, Emmanuelle Manelli, Jean-François Passegue, Carine Soulas, Bastien Venem, Claire Verdier, Véronique Youinou. 


\section{NOTES}

1. En Seine-Maritime, le Contrat de Réussite Éducative Départemental-CRED 76 est un dispositif contribuant à la réussite éducative des collégiens, en articulation avec l'Éducation nationale.

2. Organisation commune d'une conférence de Robert Halleux, historien des sciences, membre de l'Institut de France dans le cycle «Les Forums régionaux du Savoir ».

3. Site officiel : http://light2015.org/Home.html

4. À noter cependant, les objets concernés dits "patrimoniaux " présentés dans l'exposition ne sont pas juridiquement protégés, puisque les collections universitaires ne sont pas «Musée de France ", ni protégées au titre des Monuments Historiques.

\section{RÉSUMÉS}

L'utilisation du patrimoine scientifique comme outil culturel et pédagogique est à l'origine de cette expérience menée au sein de deux établissements scolaires. Les responsables du projet reviennent sur ce dispositif d'exposition - fruit d'un partenariat avec Science Action Normandie et un laboratoire de recherche - qui a permis aux élèves d'appréhender l'histoire des techniques et les métiers du patrimoine.

\section{INDEX}

Mots-clés : Patrimoine scientifique, pédagogie, scolaire

\section{AUTEURS}

\section{ANNE-SOPHIE ROZAY}

Cheffe de projet Résitech, Mission Patstec Normandie Insa Rouen Normandie anne-sophie.rozay@insa-rouen.fr

\section{SYLVAIN CHAMBRELAND}

Ingénieur de recherche en instrumentation scientifique à l'université Rouen Normandie, viceprésident du comité de pilotage Résitech et vice-président de l'université Rouen Normandie chargé de la diffusion de culture scientifique sylvain.chambreland@univ-rouen.fr 